\title{
Treatment of a Young Maxillary Central Incisor with Two Root Canals: A Case Report
}

This article was published in the following Dove Press journal:

International Journal of General Medicine

\author{
Baize Zhang' \\ Junhui Wang' \\ Zhifei Zhou ${ }^{2}$ \\ Xin $\mathrm{Ge}^{\prime}$ \\ Geng Cheng ${ }^{3}$ \\ Yujiang Chen (1)' \\ Xiaojing Wang'
}

'State Key Laboratory of Military Stomatology \& National Clinical Research Center for Oral Diseases \& Shaanxi Key Laboratory of Stomatology, Department of Pediatric Dentistry, School of Stomatology, The Fourth Military Medical University, Xi'an, 710032, People's Republic of China; ${ }^{2}$ Department of Stomatology, The General Hospital of Tibetan Military Region, Lasha, 850000 , People's Republic of China; ${ }^{3}$ State Key Laboratory of Military Stomatology \& National Clinical Research Center for Oral Diseases \& Shaanxi Key Laboratory of Stomatology, Department of Endodontics, School of Stomatology, The Fourth Military Medical University, Xi'an, 710032, People's Republic of China

Correspondence: Xiaojing Wang Department of Pediatric Dentistry, School of Stomatology, The Fourth Military Medical University, I 45 West Changle Road, Xi'an, Shaanxi, 710032,

People's Republic of China

$\mathrm{Tel} / \mathrm{Fax}$ +86-29-84776239

Email wxjingpd@hotmail.com

\begin{abstract}
Currently, the common treatment for pulpitis and periapical diseases is root canal treatment. However, the complex variations of root canal system often affect the effect of root canal treatment and even lead to treatment failure. Therefore, it is of great significance for dentists to emphasize the diversity of root canal morphology and to be familiar with the anatomical morphology of root canal to improve the success rate of clinical diagnosis and treatment. We reported a case of an eight-year-old female patient of type V root canal in a young maxillary middle incisor. The young maxillary central incisor was diagnosed with irreversible pulpitis by radiography and CBCT examination. The present case report described the entire treatment procedure of a young maxillary central incisor after trauma, including successive apical examination, CBCT examination and multiple root canal filling, and finally the treatment was completed with the aid of microscope. The root canals were filled with the vertical compression technique. Z350 composite resin was used to repair the tooth. The patient was followed-up at three, six, and 12 months after endodontic treatment. All of the examinations were normal. Finding the anatomical configuration of root canal by various ways will help to improve the success rate of root canal therapy.
\end{abstract}

Keywords: cone-beam computed tomography, CBCT, one root with two root canals, root canal microscope, young maxillary central incisor

\section{Introduction}

The objective of root canal treatment is to eliminate infection and chronic inflammation, relieve pain, and reserve the affected tooth. However, root canal treatment is often affected by many factors, one of which is the knowledge of the root canal system. Generally, we believe that the root canal system configuration of the anterior teeth, especially the maxillary central incisors, is less variant compared with that of the posterior teeth. Altman et al classified the anatomical morphology of 100 maxillary central incisors and studied the variations of their roots and root canals. They found that $100 \%$ of the teeth they studied had one root canal, ${ }^{1-3}$ and the variation in root canal morphology of the maxillary central incisors was very narrow. Most of the patients in the pediatric stomatology department who had young maxillary permanent teeth problems had chief complaints of pulpitis or periapical inflammation caused by trauma or structural abnormality. During the treatment, the apical foramen is not closed and presented as a bell mouth. Particularly, young maxillary central incisors bring more difficulties to the determination of working length and the selection of root canal filling material for root canal treatment because of their incomplete development and relatively large pulp chamber. ${ }^{4}$

The following case report presented the treatment procedure of type $\mathrm{V}$ root canal in a young maxillary middle incisor. In addition to the difficulties and challenges in the above-mentioned root canal treatment of young permanent 
teeth, one root with two root canals increases the treatment difficulty. This case report provides another possibility for the occurrence of root canal treatment failure. Through this case, we hope that dentists can carefully observe the position of the root canal and avoid omission of any root canal. If the long axis of the filling is inconsistent with the long axis of the root canal in the periapical radiograph, it indicates that there is more than one root canal.

\section{Case Report}

An eight-year-old female patient who suffered from tooth trauma half a year ago was treated in the local general hospital. Recently, she came to Department of Pediatric, School of Stomatology, the Fourth Military Medical University for treatment, with the chief complaint of recurrent swelling of the gum in the anterior teeth region and occlusal pain. Intraoral clinical and X-ray examination showed that the neck of the crown oftooth 21 was broken at the $1 / 3$ position, white filling was seen on the fracture surface, probing $(-)$, knocking $(+)$, and no obvious loosening of the tooth was found (Figure 1). Periapical radiograph showed that the apical foramen with tooth 21 was open with periapical shadow and the pulp chamber was wide.

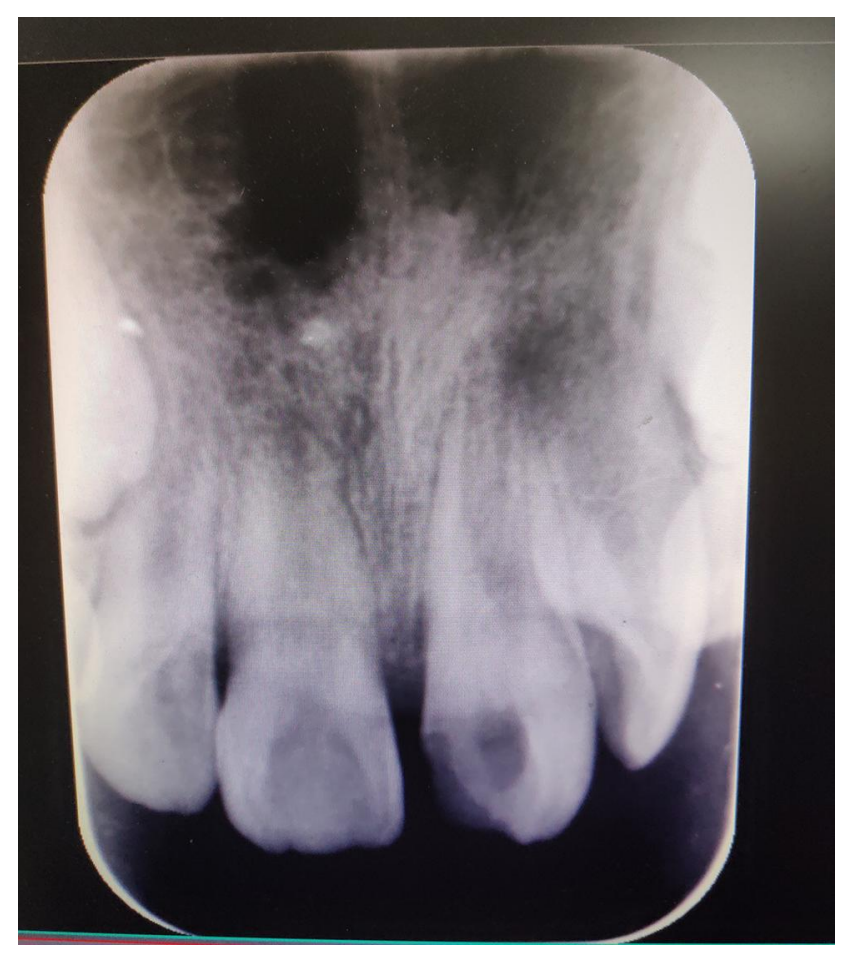

Figure I Preoperative intraoral view.
According to the treatment plan, the tooth was sterilized, triple antibiotic paste (main components were metronidazole, ciprofloxacin and minocycline) was sealed, and the status of the periapical region was observed. After the inflammation was eliminated, apexification was performed. After root development was completed at the age of ten, the root canal was treated and the defect of the tooth was repaired. The tooth will be permanently repaired when the child grows up into an adult.

At a follow-up visit, we found that the periapical shadow in the tooth gradually became smaller. Apexification was performed using Vitapex (Figure 2). Six months later, X-ray radiography showed that there was radiolucency in the root canal, the length of root increased and the apical barrier formed. Under the rubber dam isolation, the length of the root canal was measured and the root canal was prepared. The root canal was initially prepared with \#10 and \#15 K filings, then prepared with nickel-titanium instruments, and washed with $5.25 \%$ sodium hypochlorite and normal saline alternately. The root canal was filled with cold gutta-percha (Figure 3 ). During the filling of the root canal, multiple radiographs

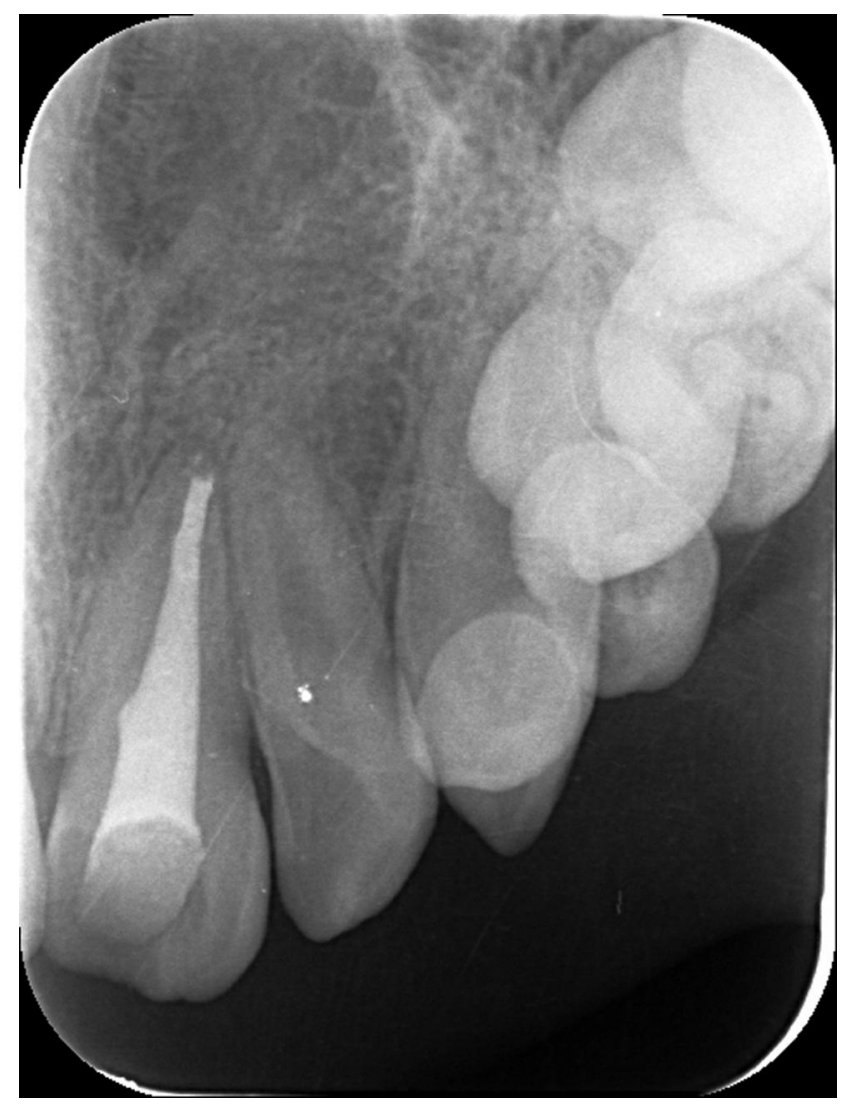

Figure 2 Apexification using vitapex in tooth 21 . 


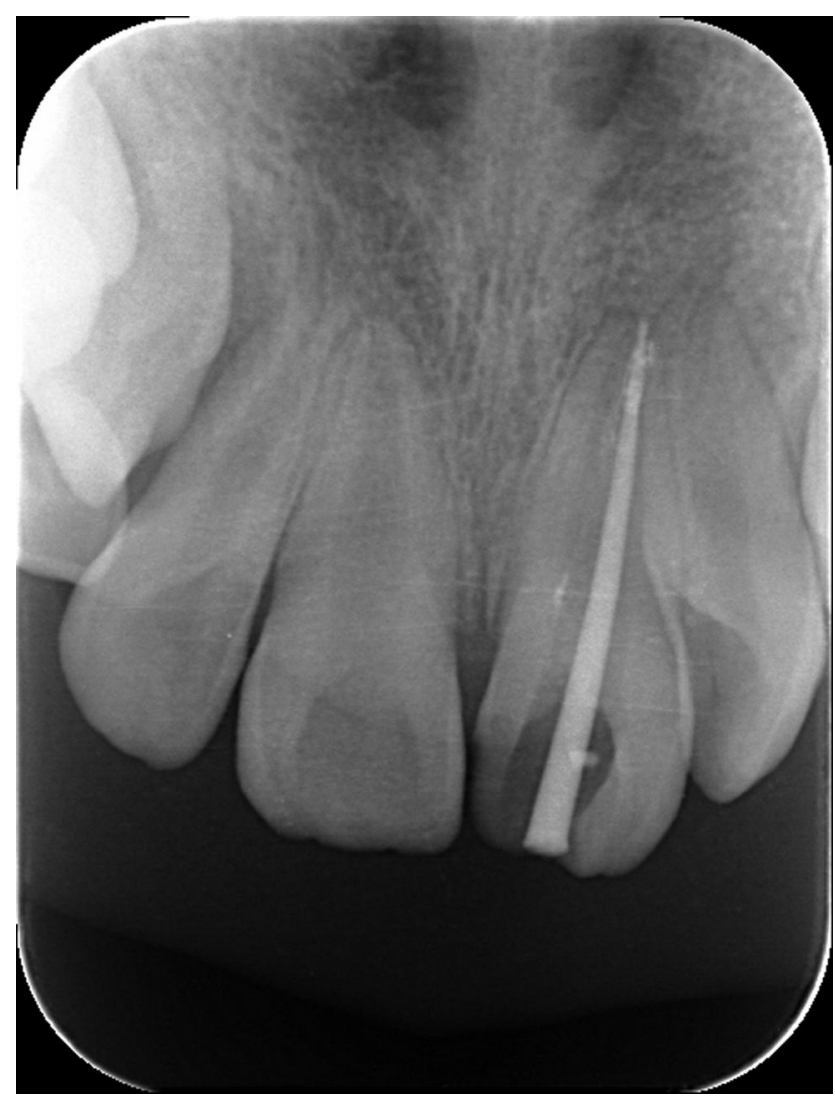

Figure 3 Working length determination radiograph of tooth 21 .

from various angles showed that radiolucency still existed in the root canal (Figure 4).

Cone-beam computed tomography (CBCT) showed that the roots were bifurcated in the middle third of the root in the mesiodistal direction, and there were two apical foramina in the apices. The root canals were explored and dredged under the microscope. We found

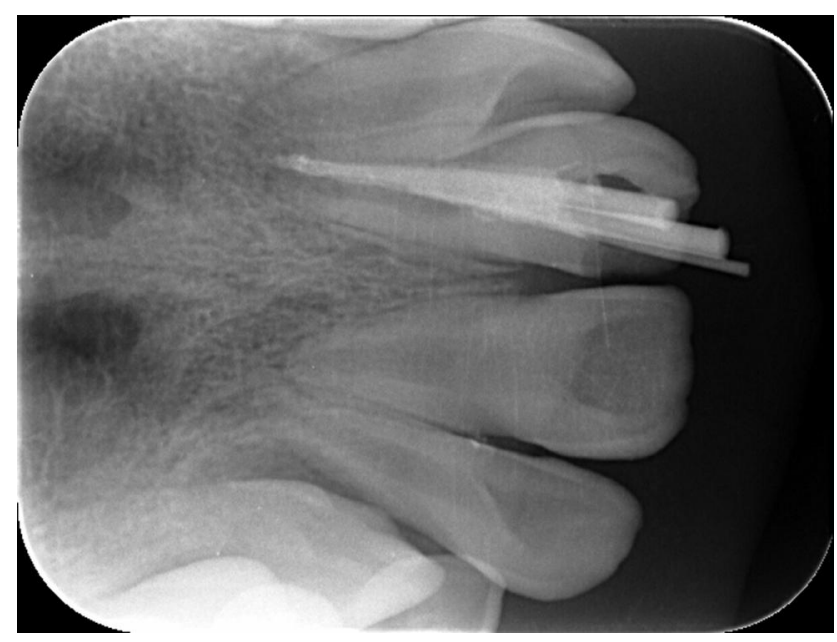

Figure 4 Filling with cold gutta-percha in tooth 21. that in the middle third of the root in the mesiodistal direction, a thin layer of canal wall divided the root canal into two independent canals. The root canals were measured again for their lengths, prepared with nickel-titanium instruments, and sealed with calcium hydroxide for two weeks before the next visit. Under the rubber dam isolation, the root canals were washed with $5.25 \%$ sodium hypochlorite and normal saline and dried, and the master gutta-percha cone was fitted. The root canals were filled with the vertical compression technique. After the operation, the quality of the filling was evaluated on a periapical radiograph (Figure 5). Finally, Z350 composite resin was used to repair the tooth.

The patient was followed-up at three, six, and 12 months after endodontic treatment. Clinical examination showed that the tooth had no percussion pain, the sinus was completely healed, gingival tissue was normal, periodontal probing depths were $2-3 \mathrm{~mm}$, and there was no abnormal loosening. Periapical radiography showed that there was no periapical radiolucency with 21 , no external absorption in the root and the periapical periodontal ligament was continuous.

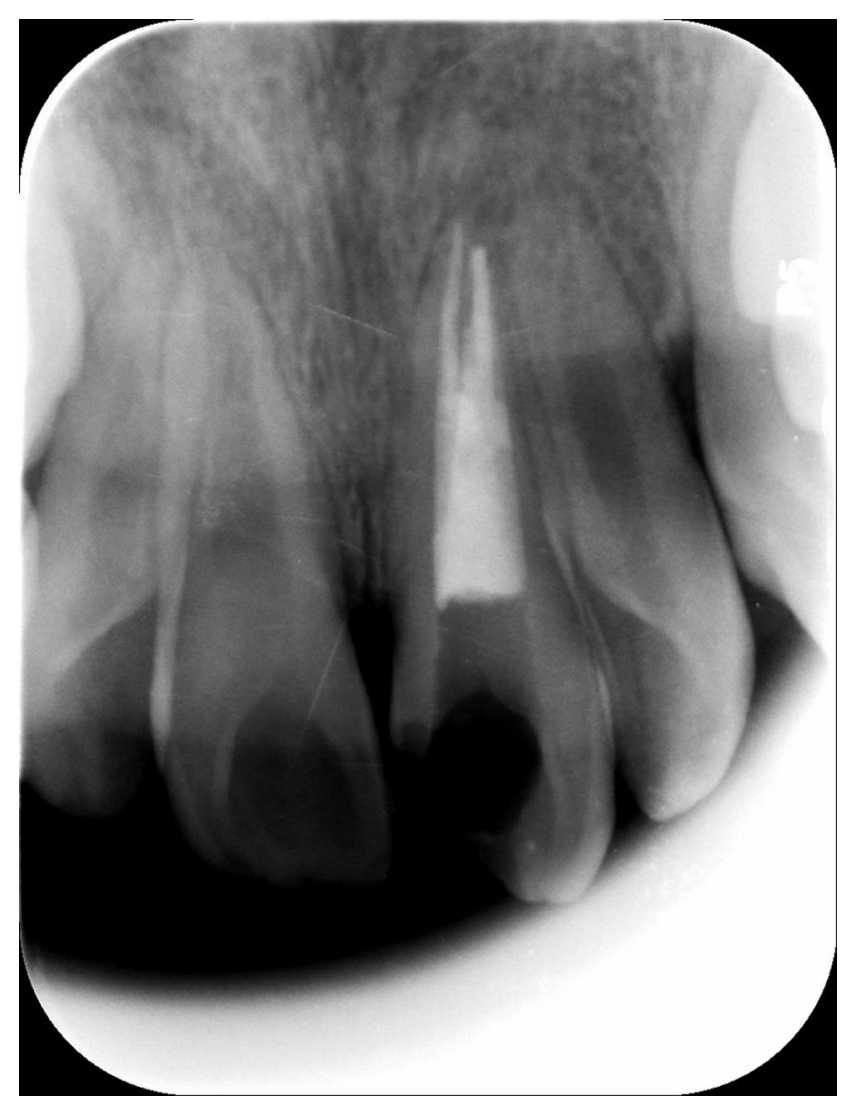

Figure 5 Radiographical view immediately after obturation of root canals. 


\section{Discussion}

The complexity and diversity of root canal system anatomy is an important factor affecting the success of root canal treatment. ${ }^{5,6}$ Nair et $\mathrm{al}^{7}$ carried out a histological examination on the apical part of posterior teeth that failed root canal treatment and found that the detection rate of bacteria in root canal isthmus and lateral branch root canal was $88 \%$. Wolcott et $\mathrm{al}^{8}$ investigated 3578 endodontically treated first molars in five years. The results showed that the incidence of MB2 canal in first molar initial root canal treatments was 58\%, and that in the nonsurgical root canal retreatments it was $66 \%$. The difference in the incidence of MB2 root canal between initial treatments and retreatments is statistically significant, which indicates that missing MB2 root canal will affect the long-term prognosis of root canal treatment. The variation in root and root canal of anterior teeth is lower compared with that of the posterior teeth. At the same time, because the root canal of young permanent teeth is wide, and apexification cannot increase the thickness of the canal wall and narrow the lumen space, which makes it more difficult to observe the root canal variation on two-dimensional images. Therefore, most dentists generally believe that maxillary central incisors only have one root and one root canal. ${ }^{1,2,9}$ We sometimes also ignored the root canal variation in the treatment of maxillary central incisor. In this case, missing a root canal is the main reason for the failure of initial root canal treatment.

As early as 1984, Vertucci classified the root canal morphology into eight types according to the number of root canal orifices and apical foramen, and the course of root canal, ${ }^{2}$ including type I (1-1 configuration), a single canal that extends from the pulp chamber to the apical foramen, with a single canal orifice and a single apical foramen; type II (2-1 configuration), two independent root canals leaving the pulp chamber and merging above the apex to form a single canal; type III (1-2-1 configuration), a single root canal leaving the pulp chamber, dividing into two canals, and subsequently merging into one canal, with the orifice in one apical foramen; type IV (2-2 configuration), two independent root canals with their orifices in two independent apical foramina; type V (1-2 configuration), a single root canal leaving the pulp chamber, and subsequently dividing into two root canals with their orifices in two apical foramina; type VI (2-1-2 configuration), two independent root canals leaving the pulp chamber, merging into one, then dividing into two canals with their orifices in two apical foramina. This case belongs to type $\mathrm{V}$ root canal. With the progress of science and technology, there are more and more techniques to study the root canal anatomy, and gradually literature reports cases of one root with more than one root canal or two roots with two root canals in maxillary central incisors. $^{10-12}$ These authors believe that it is very important to take accurate X-ray images before operation, so as to understand the general condition of the root in the affected tooth, and find out its abnormal structure and treat correspondingly through careful clinical examination. As a result, the success rate of clinical treatment can be increased.

Currently, there are many techniques to study the anatomical configuration of teeth, such as bitewing X-ray radiography, naked eye exploration, microscopic exploration of root canal, spiral CT scanning and cross-section of teeth. In this case, first of all, the root canal configuration was observed on X-ray images. We found that the pulp chamber of the tooth was wider than that of the common young permanent teeth, and the entire root canal was not in the center of the root but inclined near to the center. At the same time, radiography of the initial root canal filling showed that the distal part of the root canal was empty. Thus, we suspected that there might be a missing root canal. Because CBCT images can be used to observe the tooth in all directions through three-dimensional multiplanar reconstruction, the accuracy of CBCT images is higher than that of X-ray images. CBCT images can help to determine missing root canal more accurately. In particular, axial images can accurately display the orifice of apical foramen. Therefore, with the help of CBCT, we found that at $1 / 3$ of the root in the affected tooth, the root canal divided into two canals with two apical foramina. Because the bifurcation of the root canal was low and could not be seen with naked eyes, by the feeling of hand alone cannot guarantee the quality of cleaning and filling of the root canal. With the aid of microscope, the missing root canal was found, ${ }^{10-12}$ sterilized and filled, and the treatment was completed eventually.

The formation of the tooth root is mainly related to Hertwig's epithelial root sheath. When the root is not fully developed but the apex is infected, the development of the root can be promoted relying on the papillary cells of the root terminus or the epithelial root sheath of periapical tissue. If an extra root canal or other parts of the infected root canal system are not found, cleaned and sealed during the treatment procedure, it may cause continuous stimulation to the periapical periodontal tissue of the affected tooth, thus affecting the prognosis of root canal treatment. In this case, the sustained inflammation of the root was originally caused by the omission of a root canal in endodontic treatment. The infection of the apex was controlled using triple antibiotic paste, subsequently calcium 
hydroxide drugs were used to induce the continuous development of the root, and finally the root apex was closed. Triple antibiotic paste, originally applied to the root canal by Trope, ${ }^{4}$ can create a relatively sterile environment in the root space to allow the repair and healing of the tissue. Calcium hydroxide paste was used to guide the periapical tissue to promote the development of the root and eventually close the apical foramen.

The difficulty of root canal treatment does not lie in the treatment itself, but in the familiarity of dentists with the root canal anatomy, especially the root canal morphology of young permanent teeth. Before root canal preparation, determining the number of roots and root canals, and the general course of the root canal through various inspection techniques, further confirming the number and course of root canal with the aid of dental operating microscope, and preparing accordingly are effective measures to improve the success rate of root canal treatment. ${ }^{13,14}$

\section{Abbreviation}

CBCT, cone-beam computed tomography.

\section{Data Sharing Statement}

The data sets used and/or analyzed during this study can be obtained from Xiaojing Wang as reasonably required.

\section{Ethics Approval and Consent to Participate}

The study was approved by the Ethical Committees of the Fourth Military Medical University. Written and signed informed consent was obtained from the patient's parents to publish the case details and images.

\section{Acknowledgments}

The authors are grateful for the support from the Department of Endodontics and Pediatric Dentistry, School of Stomatology, The Fourth Military Medical University.

\section{Disclosure}

The authors report no conflicts of interest in this work.

\section{References}

1. Altman M, Guttuso J, Seidberg BH, Langeland K. Apical root canal anatomy of human maxillary central incisors. Oral Surg Oral Med Oral Pathol. 1970;30(5):694-699. doi:10.1016/0030-4220(70)90396-8

2. Vertucci FJ. Root canal anatomy of the human permanent teeth. Oral Surg Oral Med Oral Pathol. 1984;58(5):589. doi:10.1016/00304220(84)90085-9

3. Calişkan MK, Pehlivan Y, Sepetçioğlu F, Türkün M, Tuncer SS. Root canal morphology of human permanent teeth in a Turkish population. $J$ Endod. 1995;21(4):200-204. doi:10.1016/S0099-2399(06)80566-2

4. Trope M. Treatment of the immature tooth with a non-vital pulp and apical periodontitis. Dent Clin North Am. 2010;54(2):313-324. doi:10.1016/j.cden.2009.12.006

5. Peters OA. Current challenges and concepts in the preparation of root canal systems: a review. J Endod. 2004;30(8):559-567. doi:10.1097/ 01.DON.0000129039.59003.9D

6. Stropko JJ. Canal morphology of maxillary molars: clinical observations of canal configurations. $J$ Endod. 1999;25(6):446-450. doi:10.1016/S0099-2399(99)80276-3

7. Nair PN, Henry S, Cano V, Vera J. Microbial status of apical root canal system of human mandibular first molars with primary apical periodontitis after "one-visit" endodontic treatment. Oral Surg Oral Med Oral Pathol Oral Radiol Endod. 2005;99(2):231-252. doi:10.1016/j.tripleo.2004.10.005

8. Wolcott J, Ishley D, Kennedy W, Johnson S, Minnich S, Meyers J. A 5 yr clinical investigation of second mesiobuccal canals in endodontically treated and retreated maxillary molars. J Endod. 2005;31 (4):262-264. doi:10.1097/01.don.0000140581.38492.8b

9. Estrela C, Bueno MR, Couto GS, et al. Study of Root Canal Anatomy in Human Permanent Teeth in A Subpopulation of Brazil's Center Region Using Cone-Beam Computed Tomography - Part 1. Braz Dent J. 2015;26(5):530-536. doi:10.1590/0103-6440201302448

10. Maghsoudlou A, Jafarzadeh H, Forghani M. Endodontic treatment of a maxillary central incisor with two roots. J Contemp Dent Pract. 2013;14(2):345-347. doi:10.5005/jp-journals-10024-1325

11. Zaitoun H, Mackie IC. Management of a non-vital central incisor tooth with three root canals. Dent Update. 2004;31(3):142-144. doi:10.12968/denu.2004.31.3.142

12. Calvert G. Maxillary central incisor with type V canal morphology: case report and literature review. J Endod. 2014;40(10):1684-1687. doi:10.1016/j.joen.2014.01.046

13. Rampado ME, Tjäderhane L, Friedman S, Hamstra SJ. The benefit of the operating microscope for access cavity preparation by undergraduate students. J Endod. 2004;30(12):863-867. doi:10.1097/01. DON.0000134204.36894.7C

14. Buhrley LJ, Barrows MJ, BeGole EA, Wenckus CS. Effect of magnification on locating the MB2 canal in maxillary molars. $J$ Endod. 2002;28(4):324-327. doi:10.1097/00004770-200204000-00016
International Journal of General Medicine

\section{Publish your work in this journal}

The International Journal of General Medicine is an international, peer-reviewed open-access journal that focuses on general and internal medicine, pathogenesis, epidemiology, diagnosis, monitoring and treatment protocols. The journal is characterized by the rapid reporting of reviews, original research and clinical studies across all disease areas. The manuscript management system is completely online and includes a very quick and fair peer-review system, which is all easy to use. Visit http://www.dovepress.com/ testimonials.php to read real quotes from published authors. 